\title{
Hybridization of DCT and SVD in the Implementation and Performance Analysis of Video Watermarking
}

\author{
Ashish M. Kothari ${ }^{1}$ \\ ${ }^{1}$ Research Scholar, Shri Jagdishprasad Jhabarmal Tibrewala University, \\ E-mail: amkothari@aits.edu.in \\ Ved Vyas Dwivedi ${ }^{2}$ \\ ${ }^{2}$ Director \& Principal, Noble Group of Colleges, Junagadh \\ E-mail: director.principal.ngi@gmail.com
}

\begin{abstract}
In this Paper, We worked and documented the implementation and performance analysis of digital video watermarking that uses the hybrid features of two of the most powerful transform domain processing of the video and fundamentals of the linear algebra. We have taken into the account fundamentals of Discrete Cosine Transform and Singular Value Decomposition for the development of the proposed algorithm. We first used the Singular Value Decomposition and then used the singular values for the insertion of the message behind the video. Finally we used two of the visual quality matrices for the analysis purpose. We also applied various attacks on the video and found the proposed scheme more robust.
\end{abstract}

Index Terms - Digital Video Watermarking, Discrete Cosine Transform, Singular Value Decomposition, Copyright Protection, Visual Quality Matrices

\section{INTRODUCTION}

Today majority of the data transfer over the internet is in the form of the video and image and that is why there is a question of copyright protection and the proof of ownership comes into the picture every now and then. Both the task can be completed by a concept called digital watermarking which includes a number of techniques that are used to imperceptibly convey information by embedding it into the cover data [1]. In our paper we have taken video sequence as our cover data watermarking is thus called the Video Watermarking. The major factors that increases the demands of the Video watermarking [1, 2] are stated as below.

- Privacy of the digital data is required and because the copying of a video is comparatively very easy.

- Fighting against the "Intellectual property rights breach"

- Tempering of the digital video must be concealed.

- Copyright protection must not be eroded.

In this paper we concentrated on the two most useful and powerful mathematical transforms namely Discrete Wavelet Transform and the Singular Value Decomposition for the purpose of the video watermarking.

General idea of Implementation of the embedding and extraction of the watermark behind the video is explained in the next session. Discrete Wavelet Transform and Singular Value Decomposition are explained in the subsequent sessions. In Session 5, we explained various visual quality matrices which are used at the time of performance analysis. Session 6 explains the proposed algorithm. Last session focuses on the results and the conclusion part.

\section{IMPLEMENTATION}

The figure 1 shows the idea of the video watermarking at the sending end. Here first of all frames is extracted from the video sequence. The next step is to perform a color space conversion from RGB to $\mathrm{YCbCr}$ plane and divide frames into their $\mathrm{Y}, \mathrm{Cb}$ and $\mathrm{Cr}$ parts. Out of them the $\mathrm{Y}$ - luminance part is then individually given to the embedding algorithm block where the other input is a watermark to be embedded. After $\mathrm{Y}$ part is watermarked the next frame is taken and the procedure is repeated until the last frame.

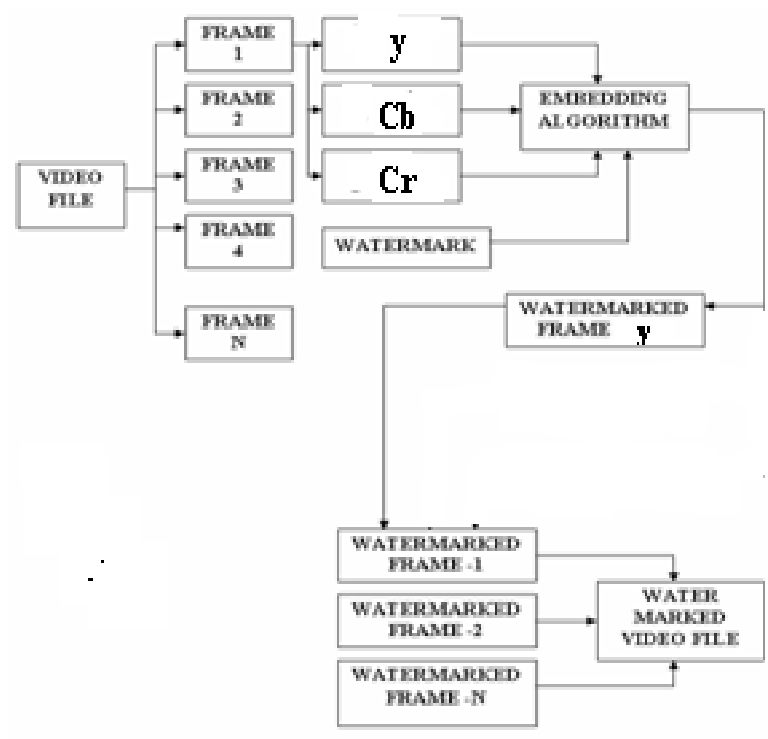

(a) 


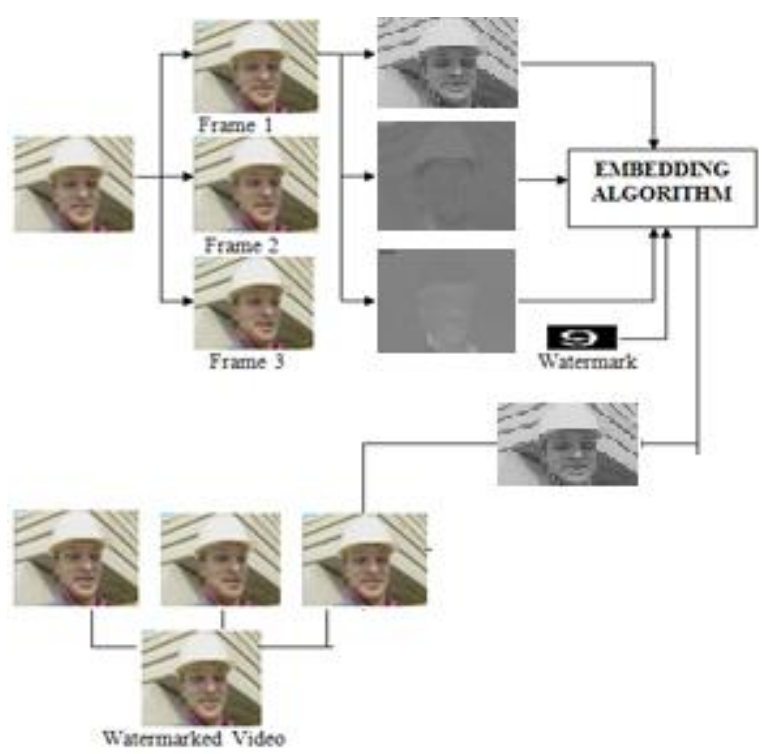

(b)

Figure 1. Idea of Video watermarking - Embedding a watermark

After the watermark is embedded in every frame, all frames are mixed to make the watermarked video which is then transmitted in the channel.

The figure 2 shows the idea of the video watermarking at the receiver end. Here watermarked video is divided into the frames. Color space conversion is performed to have $\mathrm{y}, \mathrm{Cb}$ and $\mathrm{Cr}$ parts from which $\mathrm{Y}$ part is selected and the watermark is extracted. This Procedure is repeated for all frames so as to recover all watermarks.

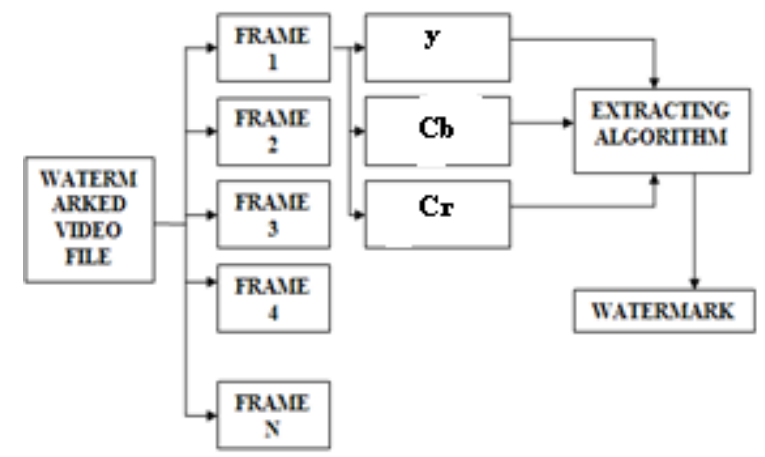

(a)

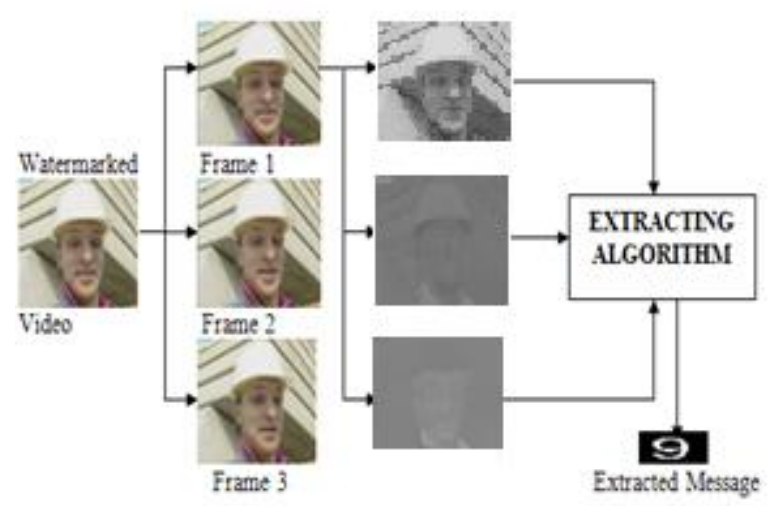

(b)

Figure 2. Idea of Video watermarking - Extracting a watermark

\section{DisCRETE COSINE TRANSFORM}

Discrete cosine transformation [4, 5, 6] (DCT) transforms a signal from the spatial into the frequency domain by using the cosine waveform. DCT concentrates the information energy in the bands with low frequency, and therefore shows its popularity in digital watermarking techniques. The DCT allows a frame to be broken up into different frequency bands, making it much easier to embed watermarking information into the middle frequency bands of a frame. The middle frequency bands are chosen such that they have minimize to avoid the most visual important parts of the frame (low frequencies) without over-exposing themselves to removal through compression and noise attacks (high frequencies).

Two dimensional DCT of a frame with size $\mathrm{MxN}$ and its inverse DCT (IDCT) are defined in Equations 1 and 2 , respectively.

$F(u, v)=$

$\alpha(u) \alpha(v) \sum_{x=0}^{M-1} \quad \sum_{y=0}^{N-1} f(x, y) \cos \left[\frac{(2 x+1) u \cdot \pi}{2 \cdot M}\right] \cos \left[\frac{(2 y+1) v \cdot \pi}{2 \cdot N}\right]$

Where $\alpha(u)=\sqrt{1 / M}$ for $\mathrm{u}=0$;

$$
\begin{array}{ll}
\alpha(u)=\sqrt{2 / M} & \text { for } \mathrm{u}=1,2,3, \ldots . \mathrm{M}-1 ; \\
\alpha(v)=\sqrt{1 / N} & \text { for } \mathrm{v}=0 ; \\
\alpha(v)=\sqrt{2 / N} & \text { for } \mathrm{v}=1,2,3, \ldots . \mathrm{N}-1 ;
\end{array}
$$

$f(x, y)=$

$\sum_{u=0}^{M-1} \quad \sum_{v=0}^{N-1} \alpha(u) \alpha(v) F(u, v) \cos \left[\frac{(2 x+1) u \cdot \pi}{2 \cdot M}\right] \cos \left[\frac{(2 y+1) v \cdot \pi}{2 \cdot N}\right]$

Where $\mathrm{x}=0,1,2, \ldots \ldots . \mathrm{M}-1, \mathrm{y}=0,1,2, \ldots \ldots . \mathrm{N}-1$

Figure 3(a) shows the three regions in the frequency domain. $F_{L}$ is used to denote the lowest frequency components of the block, while $\mathrm{F}_{\mathrm{H}}$ is used to denote the higher frequency components. $F_{M}$ is chosen as the embedding region as to provide additional resistance to lossy compression techniques, while avoiding significant modification of the cover video.

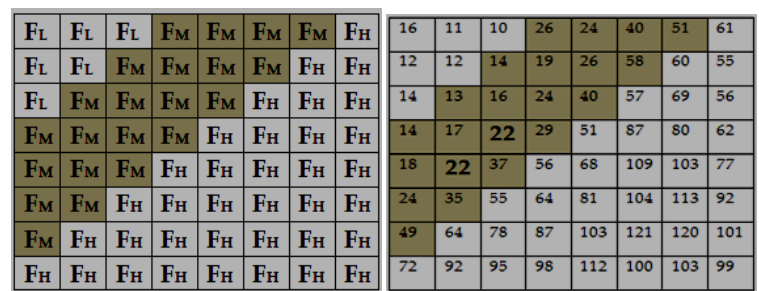
(a)

(b)

Figure 3. Defination of DCT regiouns and Quantization values used in JPEG compression scheme [3]

\section{Singular VAlue Decomposition}

Singular value decomposition (SVD) [7-11] is a numerical technique based on the linear algebra and it is used to diagonalize matrices in numerical analysis. It is an algorithm developed for a variety of applications.

When we apply SVD to an Image A of size $\mathrm{MxN}$, we find three matrices, namely $\mathrm{U}, \mathrm{V}$ and $\mathrm{S}$ whose properties are: 
- We can represent $\mathrm{A}=\mathrm{USV}^{\mathrm{T}}$.

- $\mathrm{U}$ and $\mathrm{V}$ matrices are called Unitary matrices having size $\mathrm{MxM}$ and $\mathrm{NxN}$ respectively.

- $\quad \mathrm{S}$ matrix is called diagonal matrix having size $\mathrm{MxN}$.

- The columns of the U matrix are called the left singular vectors while the columns of the $\mathrm{V}$ matrix are called the right singular vectors of $\mathrm{A}$.

- The diagonal entries of $\mathrm{S}$ are called the singular values of $\mathrm{A}$ and are arranged in decreasing order

- The singular values (SVs) of an image have very good stability, i.e., when a small perturbation is added to an image, its $\mathrm{SVs}$ do not change significantly;

- $\quad$ SVD $[12,13]$ is able to efficiently represent the intrinsic algebraic properties of an image, where singular values correspond to the brightness of the image and singular vectors reflect geometry characteristics of the image.

- An image matrix has many small singular values compared with the first singular value. Even ignoring these small singular values in the reconstruction of the image does not affect the quality of the reconstructed image [13].

An Example of the Singular Value decomposition is given as follows.

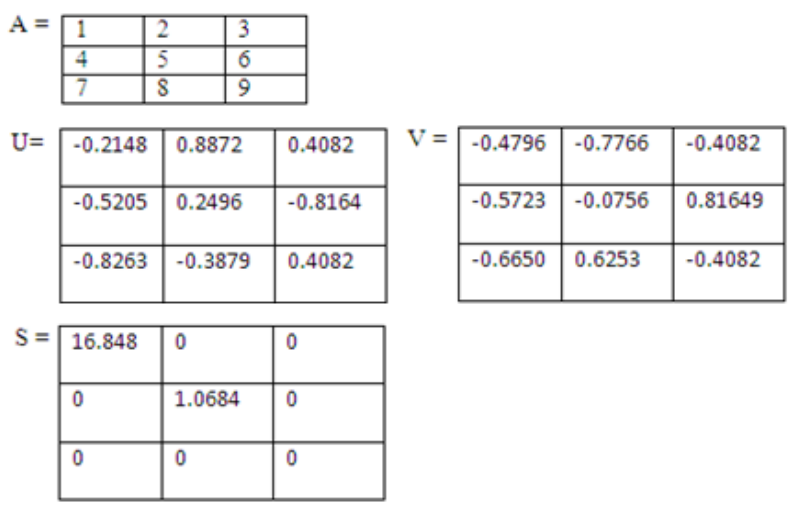

\section{VisUal QUALITY Matrices}

We have mainly used the following visual quality matrices [14] for the sake of comparison of degradation after the watermark is added to video.

$$
\begin{aligned}
& \text { MSE }=\frac{1}{M \times N} \sum_{x=1}^{M} \sum_{y=1}^{N}\left\{\left(f(x, y)-f^{\prime}(x, y)\right)^{\wedge} 2\right\} \\
& \text { PSNR }=10 \times \log \frac{255^{2}}{M S E}
\end{aligned}
$$

Here MSE - Mean Square Error

PSNR - Peak Signal to noise Ratio

$f(x, y)$ - Original Frame of the video

$\mathrm{f}^{\prime}(\mathrm{x}, \mathrm{y})$ - Watermarked Frame of the Video.

The phrase peak signal-to-noise ratio [15] , often abbreviated PSNR, is an engineering term for the ratio between the maximum possible power of a signal and the power of corrupting noise that affects the fidelity of its representation. Because many signals have a very wide dynamic range, PSNR is usually expressed in terms of the logarithmic decibel scale. A higher PSNR would normally indicate that the reconstruction is of higher quality at the receiver end.

PSNR is most easily defined via the Mean Square Error (MSE) which for two $m \times n$ Frame $f$ and $f$ ' where one of the Frames is considered a noisy approximation of the other.

\section{Proposed Algorithm}

In our proposed algorithm we have taken the foreman video as shown in the figure 4 and we have taken the message to be embedded as shown in the figure 5 .

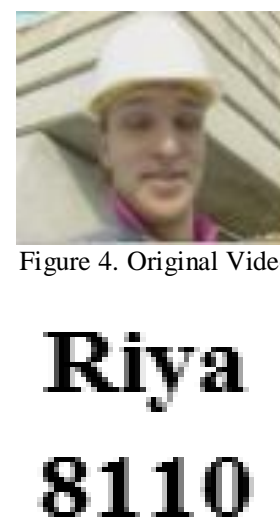

Figure 5. Message to be embedded

\section{A. EMBEDDING ALGORITHM}

Following is the stepwise representation of what we have done for the video watermarking using the proposed algorithm.

1. First of all the video is decomposed into the no. of frames.

2. Color space conversion is performed to have $\mathrm{Y}$ frame.

3. Divide the frame into the number of blocks.

4. Read the message and apply SVD to the message.

5. Take the Singular values of the SVD matrices for the embedding purpose.

6. Apply DCT to the original frame and then embed the singular values obtained in the previous step in the DC part of the DCT.

7. Apply Inverse DCT to obtain the watermarked frame.

8. Repeat step 2 to 7 for every frame.

9. Combine every watermarked frame to obtain watermarked video.

Figure 6 shows the watermarked video sequence.

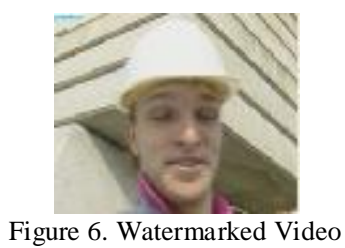

I.J. Image, Graphics and Signal Processing, 2012, 5, 14-20 
Following Table shows the results of the proposed scheme.

TABLE.1 OBTAINED PSNR AND MSE VALUES FOR FIRST FIVE FRAMES

$\begin{array}{cccc}\text { Frame No. } & \text { PSNR(DB) } & \text { MSE } & \text { Correlation } \\ 1 & 41.9171 & 11.9648 & 0.9982 \\ 2 & 41.9171 & 11.9648 & 0.9982 \\ 3 & 41.8429 & 12.1444 & 0.9974 \\ 4 & 41.8429 & 12.1444 & 0.9974 \\ 5 & 42.0171 & 11.5648 & 0.9986\end{array}$

\section{B. EXTRACTION ALGORITHM}

We followed following steps to obtain the message back from the watermarked video sequence.

1. First of all the video is decomposed into the no. of frames.

2. Color space conversion is performed to have $\mathrm{Y}$ frame.

3. Divide the frame into the number of blocks.

4. Apply DCT to the watermarked frame.

5. Obtain the singular values from the blocks.

6. Apply inverse SVD to find out the watermark bits.

7. Repeat steps 2 to 5 for extracting the watermarks from all the frames.

Following are the extraction results what we got from Y frame without attack.

\section{Riya 8110}

Figure 7. Message Extracted from Y Frame without any attack

\section{RESULTS}

We applied various attacks on the video and found various results as discussed below.

\section{A. COMPRESSION ATTACK}

We passed the video from the jpeg compression attack and achieved results as shown in the table 2 .

TABLE. 2 OBTAINED RESULTS UNDER COMPRESSION ATTACK

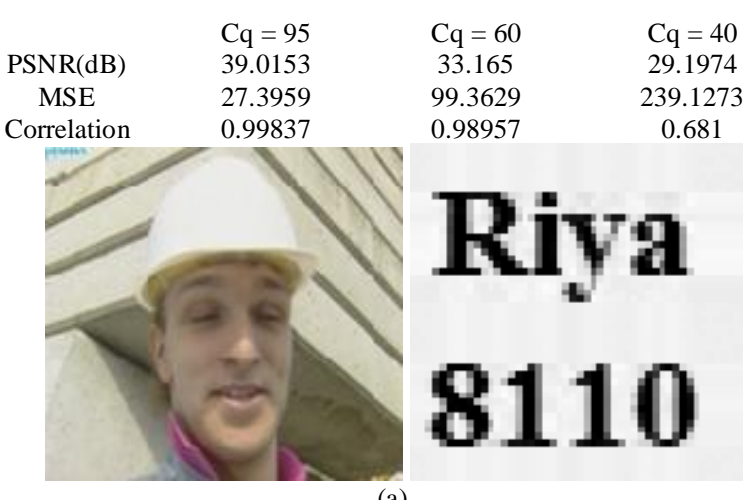

(a)
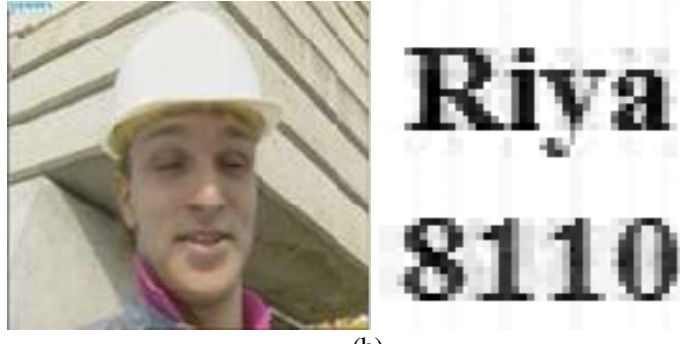

(b)

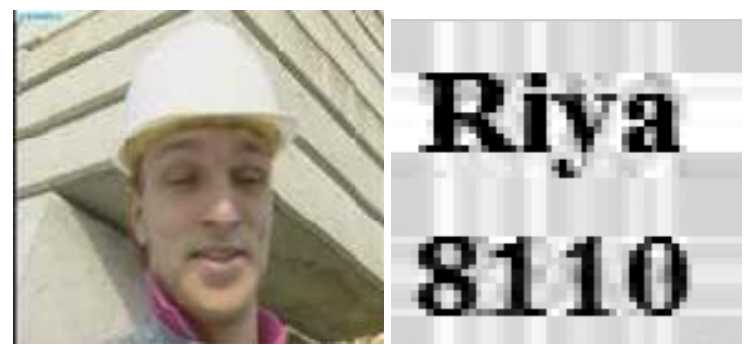

(c)

Figure 8. Watermarked Frame and messages extracted under compression attack with (a) $\mathrm{CQ}=95$ (b) $\mathrm{CQ}=60$ (c) $\mathrm{CQ}=40$

\section{B. GAUSSIAN LPF ATTACK}

Table 3 shows the results of Gaussian LPF with a mask size of $3 \times 3$ and various sigma.

TABLE.3 OBTAINED RESULTS UNDER GAUSSIAN LPF ATTACK

$\begin{array}{cccc} & \text { Sigma }=0.5 & \text { Sigma }=2 & \text { Sigma }=3.5 \\ \text { PSNR(dB) } & 34.4421 & 27.2207 & 27.0077 \\ \text { MSE } & 64.7254 & 333.9381 & 350.4674 \\ \text { Correlation } & 0.96338 & 0.83254 & 0.8129\end{array}$

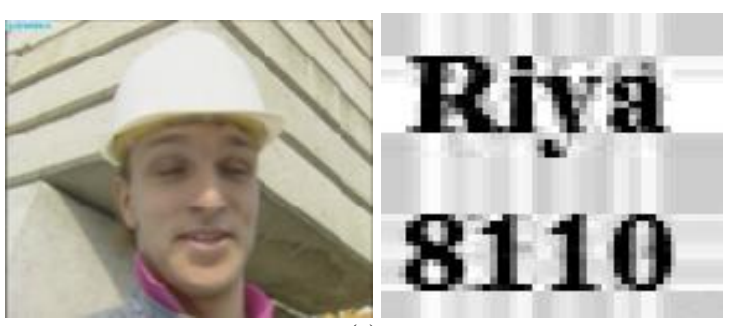

(a)

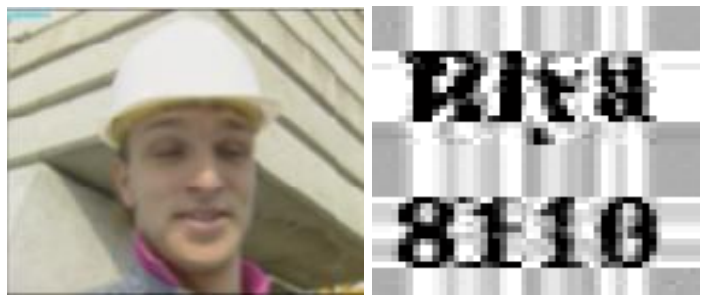

(b)

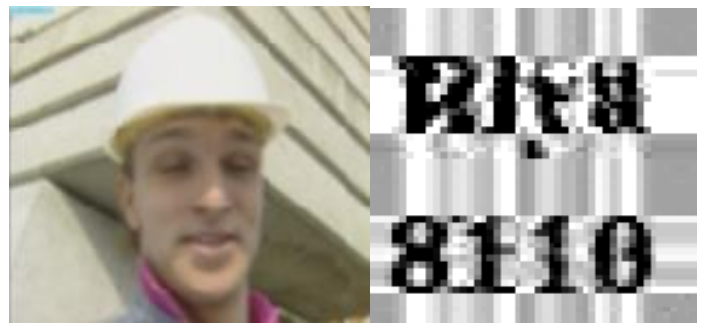

(c)

Figure 9. Watermarked Frame and messages extracted under gaussian LPF attack with (a) sigma $=0.5$ (b) sigma $=2$ (c) sigma $=3.5$ 


\section{GAUSSIAN NOISE ATTACK}

We added Gaussian noise with 0 mean various variances as described in the table 4 .

TABLE.4 OBTAINED RESULTS UNDER GAUSSIAN NOISE ATTACK

$\begin{array}{cccc} & \text { Variance }= & \text { Variance }= & \text { Variance }=0.01 \\ & 0.0005 & 0.005 & \\ \text { PSNR(dB) } & 39.0153 & 33.165 & 29.1974 \\ \text { MSE } & 27.3959 & 99.3629 & 239.1273 \\ \text { Correlation } & 0.99837 & 0.98957 & 0.681\end{array}$

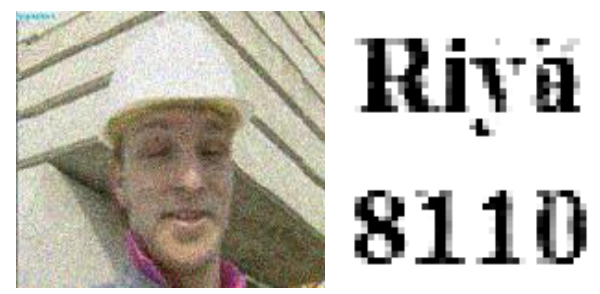

(a)
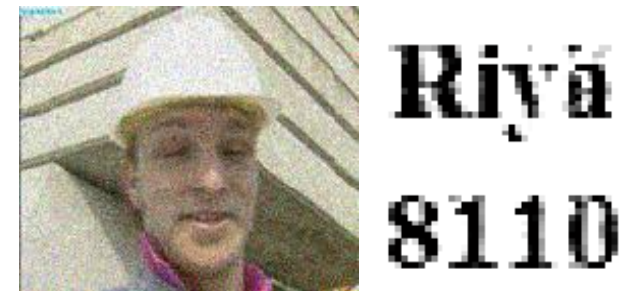

(b)
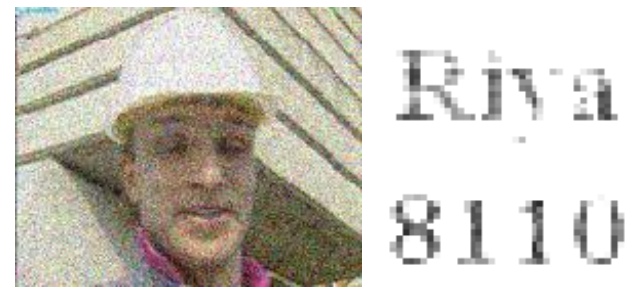

(c)

Figure 10. Watermarked Frame and messages extracted under gaussian noise attack with Variance (a) 0.0005 (b) 0.005 (c) 0.01

\section{SALT \& PEPPER NOISEATTACK}

We added salt $\&$ pepper noise with various variances and got results as per table 5 .

TABLE.5 OBTAINEd Results Under SAlt \& PEPPER NoISE ATt ACK

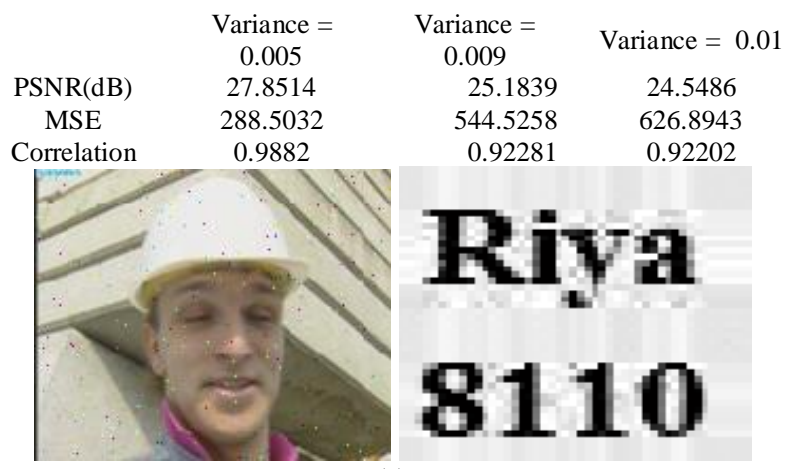

(a)
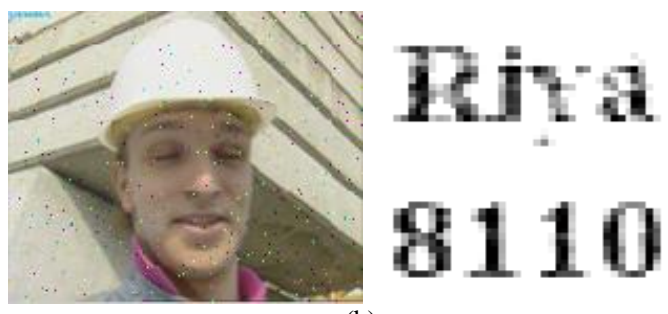

(b)
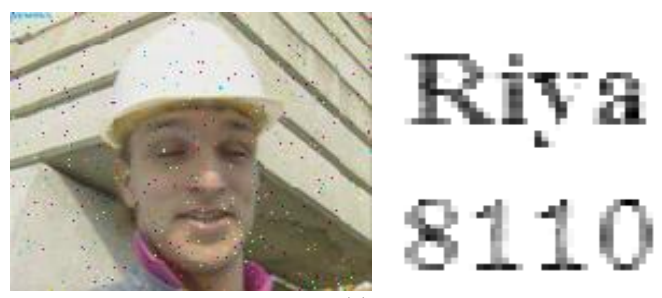

(c)

Figure 11. Watermarked Frame and messages extracted under salt \& pepper noise attack with Variance (a) 0.0005 (b) 0.009 (c) 0.01

\section{E. SPACKLE NOISEATTACK}

We applied Spackle noise attack to the frame with various variances and taken results which appears in table 6.

TABle.6 OBTAined Results UndER SPACKLE NOISE ATTACK

$\begin{array}{cccr} & \text { Variance }= & \text { Variance }= & \text { Variance }=0.01 \\ & 0.005 & 0.009 & 23.1421 \\ \text { PSNR(dB) } & 26.0079 & 23.5772 & 814.2854 \\ \text { MSE } & 421.7581 & 736.2925 & 0.88243 \\ \text { Correlation } & 0.98259 & 0.93392 & \end{array}$

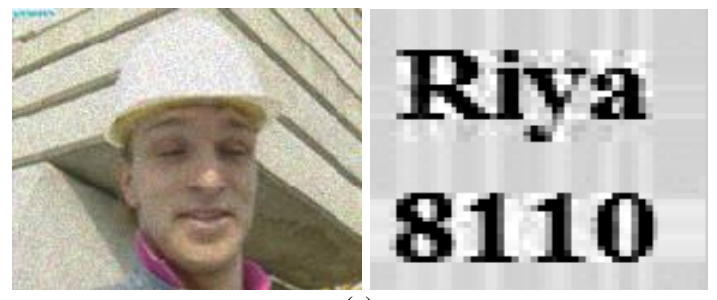

(a)

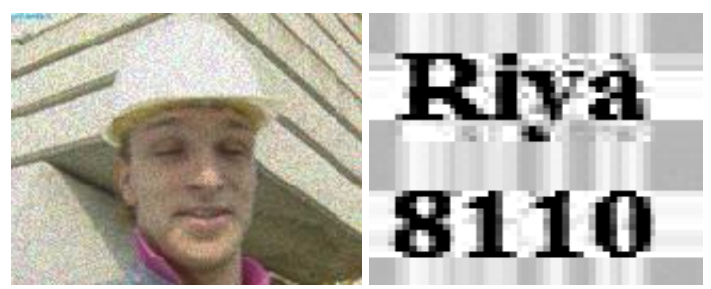

(b)

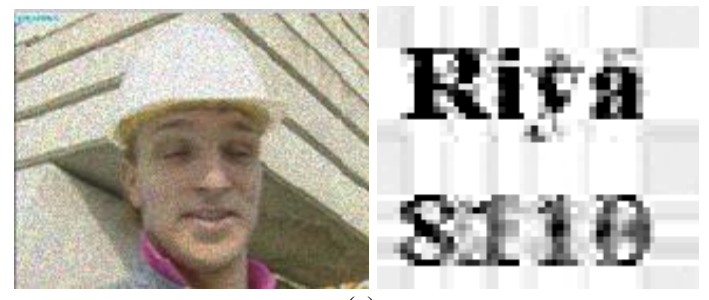

(c)

Figure 12. Watermarked Frame and messages extracted under spackle noise attack with Variance (a) 0.0005 (b) 0.009 (c) 0.01 


\section{CONCLUSION}

From the work done we concluded proposed scheme is robust against compression, Gaussian LP filtering, Gaussian noise, Salt \& Pepper noise and Spackle noise. We also concluded that this scheme fails in average filtering, median filtering, motion blur, rotation and intensity adjustment attacks.

\section{REFERENCES}

[1] Debnath Bhattacharyya. "Watermarking Using Multiresolution Cosine Transformation: A Review", Communications in Computer and Information Science, 2011.

[2] Huang D., Liu J., Huang J., “A DWT-based image watermarking algorithm", Proc. IEEE Intl. Conf. Multimedia and Expo., pp.429-432, 2001.

[3] Chiou-Ting Hsu and Ja-Ling Wu, "DCT-BASED WATERMARKING FOR VIDEO”, IEEE Transactions on Consumer Electronics, Vol. 44, No. 1, FEBRUARY 1998

[4] E. Koch and J. Zhao, "Towards Robust and Hidden Image Copyright Labeling," Proc. IEEE Nonlinear Signal and Image Processing, pp. 452-455, June 1995.

[5] C. T. Hsu and J. L. Wu, "Hidden Signatures in Images," Proceeding of International Conference on Image Processing, pp. 223-226, Sep. 1996.

[6] I. J. Cox, J. Kilian, T. Leighton and T. Shamoon, "Secure Spread Spectrum Watermarking for Multimedia," Tech. Rep. 95-1 0, NEC Research Institute, 1995.

[7] H. C. Andrews and C. L. Patterson, "Singular Value Decomposition (SVD) image coding" IEEE Trans. on Communications, pp. 425-432, April 1976.

[8] G. H. Golub and C. Reinsch, "Singular value decomposition and least squares solutions", Numer. Math., Vol. 14, pp. 403-420, 1970.

[9] Liu, R., and T. Tan, 2002. "A SVD-Based Watermarking Scheme for Protecting Rightful Ownership", IEEE Trans. Multimedia 4, pp.121128.

[10] Chang, C., P.Tsai, and C. Lin, 2005. "SVD-based digital image watermarking scheme", Pattern Recognition Letters 26, pp.1577-1586.

[11] Wu, Y, 2005. "On the Security of SVD-Based Ownership Watermarking", IEEE Trans. Multimedia 7, pp. 624-627.

[12] Ke-feng he, et al , "Watermarking for images using the HVS and SVD in the wavelet domain" Proceedings of IEEE International Conference on Mechatronics and Automation, pp.2352- 2356, 2006

[13] V.Santhi and Dr. Arunkumar Thangavelu, "DWTSVD Combined Full Band Robust Watermarking Technique for Color Images in YUV Color Space", International Journal of Computer Theory and Engineering, Vol. 1, No. 4, October2009.
[14] M. Kutter \& F.A.P.Petitcolas, "A fair benchmark for image watermarking systems", Electronic Imaging '99. Security and Watermarking of Multimedia Contents, vol. 3657, Sans Jose, CA, USA, 25-27 January 1999.

[15] Web page http://en.wikipedia.org/wiki/Peak_signal-tonoise_ratio

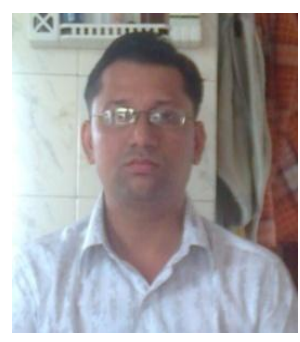

Mr. Ashish M. Kothari has completed his bachlor and masters degree in the Electronics \& Communication Engineering from saurashtra university, rajkot, gujarat, india and he is currenly pursuing his $\mathrm{Ph}$. D. in the same discipline from shree jagdishprasad jhambarmal tibrewal university, jhunjhunu, rajsthan, india. His Area of reaserch is to design a robust method for the video watermarking. He is currently working as an assistant professor in the department of electronics \& communication engineering at Atmiya Institute of technology \& science, rajkot, gujarat, india. $\mathrm{He}$ is also appointed as a director of the UDISHA club by Gujarat Technological University for the rajkotveraval sankul 1. He is also appointed as PG coordinator for the masters program in $\mathrm{EC}$ in the department of Electronics \& Communication at Atmiya Institute of technology \& science, rajkot, gujarat, india. He has guided more than $20 \mathrm{UG}$ students in their project work and more than 7 students in their dissertation work at PG level. He has presented 12 national and international papers and published 4 international papers. He has published a book titled real time anlysis of digital image watermarking with Lambart Publishing house, Germany.

His area of interest is Image and video processing, microcontrollers and embedded systems.

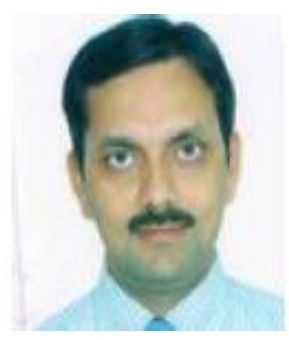

Professor (Dr) Ved Vyas Dwivedi is a B.E.(EC), M.E.(EC), and Ph.D. (EC). He worked with Tata Chemicals Ltd., Elecon Engg. Co. Ltd. and Valcan Engg. Co. Ltd. in various capacities starting from G.E.T., Jr Engr, and Sr R \& d Engr. He worked in CIT Changa and Charusat as assistant professor and associate professor and is currently working as professor and Director in Noble Group of Institutios Junagadh. He is life member of IETE, IE, ISTE and member IEEE (2003, '04, '09, '10) alongwith MTTS. $\mathrm{He}$ has been offering his services as reviewer of various national and international Journals such as Doves - Journal of Nano technology and Sciences, 
Journal of Computer Engineering, Journal of Electronics researches, and reviewer of various international and national conferences sponsored by IEEE, IETE and IE. He is guiding five $\mathrm{Ph} \mathrm{D}$ candidates and has guided $23 \mathrm{M}$. Tech. dissertations. He has published 28 Journal papers and 42 Conference papers. He has co-authored a book on microwave engineering and co-chair of Junagadh-GTU Innovation Sankul.

His Fields of interest and research are wirelessoptical-mobile-satellite communication, radar microwave-electromagnetic-antenna-RF engineering and Metamaterials for passive components and signal and image processing where he is currently guiding two of his four fellows. 